2019 4th International Conference on Information Technology, Information Systems and Electrical Engineering (ICITISEE)

EICITISEE

took place 20-21 November 2019 in Yogyakarta, Indonesia.

$\begin{array}{ll}\text { IEEE catalog number: } & \text { CFP19G48-ART } \\ \text { ISBN: } & 978-1-7281-5118-2\end{array}$

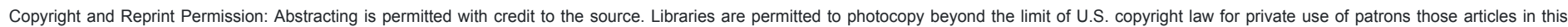

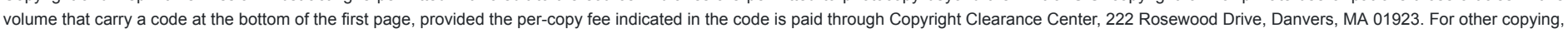
reprint or republication permission, write to IEEE Copyrights Manager, IEEE Operations Center, 445 Hoes Lane, Piscataway, NJ 08854 . All rights reserved. Copyright $\odot 2019$ by IEEE. 


\section{General Chair}

Kusrini Kusrini (AMIKOM Yogyakarta University, Indonesia)

\section{Publication Chair}

Sumarni Adi (Universitas AMIKOM Yogyakarta, Indonesia)

Akhmad Dahlan (Universitas Amikom Yogyakarta, Indonesia)

\section{Secretary}

Yoga Pristyanto (Universitas Amikom Yogyakarta, Indonesia)

Nila Puspitasari (Universitas AMIKOM Yogyakarta, Indonesia)

\section{Treasurer}

Sumarni Adi (Universitas AMIKOM Yogyakarta, Indonesia)

\section{Chair of Technical Program Committee}

Emha Taufiq Luthfi (Universitas AMIKOM Yogyakarta, Indonesia)

Lesnanto Multa Putranto (UGM, Indonesia)

Arief Setyanto (Universitas AMIKOM Yogyakarta, Indonesia)

Andi Sunyoto (Universitas AMIKOM Yogyakarta, Indonesia)

Ferry Wahyu Wibowo (Universitas Amikom Yogyakarta, Indonesia) 


\section{Technical Program Committee}

\begin{tabular}{|c|c|c|}
\hline Intan Ermahani A. Jalil & Universiti Teknikal Malaysia Melaka & Malaysia \\
\hline Mohd Helmy Abd Wahab & Universiti Tun Hussein Onn Malaysia & Malaysia \\
\hline MS. Hendriyawan Achmad & Yogyakarta University of Technology & Indonesia \\
\hline Sumarni Adi & Universitas AMIKOM Yogyakarta & Indonesia \\
\hline I Adiyasa & Gadjah Mada University & Indonesia \\
\hline I Made Agastya & Universitas Amikom Yogyakarta & Indonesia \\
\hline Mohd Khairul Ikhwan Ahmad & Universiti Tun Hussein Onn Malaysia & Malaysia \\
\hline Ali Qusay Al-Faris & Assumption College & USA \\
\hline Shajith Ali & SSN College of Engineering, Chennai & India \\
\hline Gede Angga Pradiptha & Institut Teknologi dan Bisnis STIKOM Bali & Indonesia \\
\hline Igi Ardiyanto & Universitas Gadjah Mada & Indonesia \\
\hline Dhani Ariatmanto & Universitas Amikom Yogyakarta & Indonesia \\
\hline Agus Aribowo & Universitas Pembangunan Nasional Veteran Yogyakarta & Indonesia \\
\hline Andria Arisal & Indonesian Institute of Sciences & Indonesia \\
\hline Arti Arya & PESIT-Bangalore South Campus & India \\
\hline Ika Astuti & Universitas Amikom Yogyakarta & Indonesia \\
\hline Hasyim Asyari & Universitas Muhammadiyah Surakarta & Indonesia \\
\hline Azizul Azizan & Universiti Teknologi Malaysia (UTM) & Malaysia \\
\hline Aslina Baharum & Universiti Malaysia Sabah & Malaysia \\
\hline Aashish Bardekar & Sipna College of Engineering and Technology, Amravati & India \\
\hline Agus Bejo & Universitas Gadjah Mada & Indonesia \\
\hline Adha Imam Cahyadi & Universitas Gadjah Mada & Indonesia \\
\hline Alessandro Carrega & CNIT & Italy \\
\hline Maria Chiara Caschera & CNR & Italy \\
\hline Selem Charfi & HD Technology & France \\
\hline Wichian Chutimaskul & King Mongkut's University of Technology Thonburi & Thailand \\
\hline Domenico Ciuonzo & University of Naples Federico II, IT & Italy \\
\hline Akhmad Dahlan & Universitas Amikom Yogyakarta & Indonesia \\
\hline
\end{tabular}




\begin{tabular}{|c|c|c|}
\hline Ni Ketut Dewi Ari Jayanti & Institute of Technology and Business STIKOM Bali & Indonesia \\
\hline Andi Wahju Rahardjo Emanuel & Universitas Atma Jaya Yogyakarta & Indonesia \\
\hline Ahmad Fajar & Bina Nusantara University & Indonesia \\
\hline Muhammad Faris & Universitas Gadjah Mada & Indonesia \\
\hline Hanif Fatta & Universitas AMIKOM Yogyakarta & Indonesia \\
\hline Anggit Ferdita Nugraha & Universitas AMIKOM Yogyakarta & Indonesia \\
\hline Alireza Ghasempour & ICT Faculty & USA \\
\hline Arifiyanto Hadinegoro & Amikom Universty Yogyakarta & Indonesia \\
\hline Byeong-jun Han & Soongsil University & Korea (South) \\
\hline Muh Hanafi & Universitas Amikom Yogyakarta & Indonesia \\
\hline Seng Hansun & Universitas Multimedia Nusantara & Indonesia \\
\hline Hartatik Hartatik & STMIK AMIKOM Yogyakarta & Indonesia \\
\hline Mardhiya Hayaty & Universitas AMIKOM Yogyakarta & Indonesia \\
\hline Purwono Hendradi & Universitas Muhammadiyah Magelang & Indonesia \\
\hline Roberto Carlos Herrera Lara & National Polytechnic School & Ecuador \\
\hline Indriana Hidayah & Universitas Gadjah Mada & Indonesia \\
\hline Tonny Hidayat & Universitas AMIKOM Yogyakarta & Indonesia \\
\hline Nor Hikmah & Universitas AMIKOM & Indonesia \\
\hline Danial Hooshyar & Korea University & Korea (South) \\
\hline Hozairi Hozairi & Islamic University of Madura & Indonesia \\
\hline Imelda Imelda & Universitas Budi Luhur & Indonesia \\
\hline Rini Indrayani & Universitas Amikom Yogyakarta & Indonesia \\
\hline Nurulisma Ismail & Universiti Malaysia Perlis & Malaysia \\
\hline Iswandi Iswandi & Gadjah Mada University & Indonesia \\
\hline Ramkumar Jaganathan & VLB Janakiammal College of Arts and Science & India \\
\hline Arihant Jain & Jaipur Engineering College \& Research Centre & India \\
\hline Biao Jiang & The City University of New York & USA \\
\hline Dimitrios Kallergis & University of West Attica & Greece \\
\hline Sandy Kosasi & STMIK Pontianak & Indonesia \\
\hline
\end{tabular}




\begin{tabular}{|c|c|c|}
\hline Krisnawati Krisnawati & University of AMIKOM Yogyakarta & Indonesia \\
\hline Ryan Kristianto & Universitas Amikom Yogyakarta & Indonesia \\
\hline Sumit Kushwaha & KNIT Sultanpur & India \\
\hline Kusnawi Kusnawi & AMIKOM University & Indonesia \\
\hline Kusrini Kusrini & AMIKOM Yogyakarta University & Indonesia \\
\hline Pavel Loskot & Swansea University & United Kingdom (Great Britain) \\
\hline Emha Taufiq Luthfi & Universitas AMIKOM Yogyakarta & Indonesia \\
\hline Mahdin Mahboob & Stony Brook University & USA \\
\hline Robert Marco & STMIK Amikom Yogyakarta & Indonesia \\
\hline Prita Dewi Mariyam & Universitas Indonesia & Indonesia \\
\hline Ahlihi Masruro & Universitas AMIKOM Yogyakarta & Indonesia \\
\hline Ratheesh Kumar Meleppat & University of California Davis & USA \\
\hline Ahmed Mobashsher & The University of Queensland & Australia \\
\hline Seyed Sahand Mohammadi Ziabari & Vrije University of Amsterdam & The Netherlands \\
\hline Amrit Mukherjee & Jiangsu University & China \\
\hline I Wayan Mustika & Universitas Gadjah Mada & Indonesia \\
\hline Syibrah Naim & Universiti Sains Malaysia & Malaysia \\
\hline Warsun Najib & Universitas Gadjah Mada & Indonesia \\
\hline Asro Nasiri & University of Amikom Yogyakarta & Indonesia \\
\hline Shah Nazir & University of Peshawar & Pakistan \\
\hline Ruzelita Ngadiran & Universiti Malaysia Perlis & Malaysia \\
\hline Bhanu Nugraha & Universitas Amikom Yogyakarta & Indonesia \\
\hline Hanung Nugroho & Universitas Gadjah Mada & Indonesia \\
\hline Prapto Nugroho & Universitas Gadjah Mada & Indonesia \\
\hline Nitish Ojha & DIT University, Dehradun & India \\
\hline Ilker Ali Ozkan & Selcuk University & Turkey \\
\hline Oktavia Permata & Institut Teknologi Telkom Surabaya & Indonesia \\
\hline Anugerah Persada & Universitas Gadjah Mada & Indonesia \\
\hline Kiran Sree Pokkuluri & Shri Vishnu Engineering College for Women & India \\
\hline
\end{tabular}




\begin{tabular}{|c|c|c|}
\hline N. Prabaharan & SASTRA Deemed University & India \\
\hline Gede Pramudya Ananta & Universiti Teknikal Malaysia Melaka & Malaysia \\
\hline Andri Pranolo & Universitas Ahmad Dahlan & Indonesia \\
\hline Prihandoko Prihandoko & University of Gunadarma & Indonesia \\
\hline Yoga Pristyanto & Universitas Amikom Yogyakarta & Indonesia \\
\hline Reza Pulungan & Universitas Gadjah Mada & Indonesia \\
\hline Nila Puspitasari & Universitas AMIKOM Yogyakarta & Indonesia \\
\hline Guntur Putra & University of New South Wales & Australia \\
\hline Lesnanto Multa Putranto & UGM & Indonesia \\
\hline Ali Rafiei & University of Technology Sydney & Australia \\
\hline Suwanto Raharjo & Informatics of The Institut Sains \& Teknologi AKPRIND Yogyakarta & Indonesia \\
\hline Hemant Kumar Rath & Tata Consultancy Services & India \\
\hline Rizky Rizky & Universitas AMIKOM Yogyakarta & Indonesia \\
\hline Rumini Rumini & Universitas AMIKOM Yogyakarta & Indonesia \\
\hline G. p. Sajeev & Amrita Vishwa Vidyapeetham & India \\
\hline Andreas Sandiwan & Gadjah Mada University & Indonesia \\
\hline Banu Santoso & Universitas AMIKOM Yogyakarta & Indonesia \\
\hline Leo Santoso & Petra Christian University & Indonesia \\
\hline Sayantam Sarkar & Vijaya Vittala Institute of Technology & India \\
\hline Theopilus Bayu Sasongko & Universitas AMIKOM Yogyakarta & Indonesia \\
\hline Mithileysh Sathiyanarayanan & City, University of London & United Kingdom (Great Britain) \\
\hline Vaibhav Saundarmal & Marathwada Institute of Technology, Aurangabad & India \\
\hline Enny Sela & Universitas Teknologi Yogyakarta & Indonesia \\
\hline Erni Seniwati & Universitas AMIKOM Yogyakarta & Indonesia \\
\hline Anindita Septiarini & Univeristas Mulawarman & Indonesia \\
\hline Amel Serrat & USTO MB & Algeria \\
\hline Bayu Setiaji & Universitas AMIKOM Yogyakarta & Indonesia \\
\hline Noor Akhmad Setiawan & Universitas Gadjah Mada & Indonesia \\
\hline Emy Setyaningsih & Institute of Science \& Technology AKPRIND & Indonesia \\
\hline
\end{tabular}




\begin{tabular}{|c|c|c|}
\hline Arief Setyanto & Universitas AMIKOM Yogyakarta & Indonesia \\
\hline Iwan Setyawan & Satya Wacana Christian University & Indonesia \\
\hline Andik Setyono & Universitas Dian Nuswantoro & Indonesia \\
\hline Aditi Sharma & Quantum University, Roorkee, Uttarakhand & India \\
\hline Vesh Raj Sharma Banjade & Intel Corporation & USA \\
\hline Abdul Samad Shibghatullah & UCSI University & Malaysia \\
\hline Monika Sikri & Cisco Systems India Pvt Ltd. & India \\
\hline Amando Singun & Higher College of Technology, Muscat & Oman \\
\hline Achmad Solichin & Universitas Budi Luhur & Indonesia \\
\hline lickho Song & Korea Advanced Institute of Science and Technology & Korea (South) \\
\hline Yi-Jen Su & Shu-Te University & Taiwan \\
\hline Suhirman Suhirman & Universitas Teknologi Yogyakarta & Indonesia \\
\hline Andi Sunyoto & Universitas AMIKOM Yogyakarta & Indonesia \\
\hline Sutarman Sutarman & Faculty Of Information Technology and Business, University Technoloy of Yogyakarta & Indonesia \\
\hline Melwin Syafrizal & Universitas AMIKOM Yogyakarta & Indonesia \\
\hline Srinivasulu Tadisetty & Kakatiya University College of Engineering and Technology & India \\
\hline Chakib Taybi & Mohammed First University & Morocco \\
\hline Ivanna Timotius & Satya Wacana Christian University & Indonesia \\
\hline Evi Triandini & Institut Teknologi dan Bisnis STIKOM Bali & Indonesia \\
\hline Hastari Utama & Universitas Amikom Yogyakarta & Indonesia \\
\hline Ema Utami & Universitas Amikom Yogyakarta & Indonesia \\
\hline Dario Vieira & EFREI & France \\
\hline Anik Vitianingsih & Universitas Dr Soetomo & Indonesia \\
\hline Mochammad Wahyudi & Universitas Gadjah Mada & Indonesia \\
\hline Sri Ngudi Wahyuni & Universitas AMIKOM Yogyakarta & Indonesia \\
\hline Fikri Waskito & Universitas Gadjah Mada & Indonesia \\
\hline Leong Wen Chek & University of Malaya & Malaysia \\
\hline Sunu Wibirama & Universitas Gadjah Mada & Indonesia \\
\hline Adi Wibowo & Diponegoro University & Indonesia \\
\hline
\end{tabular}




\begin{tabular}{|c|c|c|}
\hline Ferry Wahyu Wibowo & Universitas Amikom Yogyakarta & Indonesia \\
\hline Sigit Wibowo & Gadjah Mada University & Indonesia \\
\hline Teguh Wibowo & Gadjah Mada University & Indonesia \\
\hline Ainul Yaqin & Universitas Amikom Yogyakarta & Indonesia \\
\hline Thaweesak Yingthawornsuk & King Mongkut's University of Technology Thonburi & Thailand \\
\hline Uky Yudatama & Universitas Indonesia & Indonesia \\
\hline Go Yun II & Heriot-Watt University Malaysia & Malaysia \\
\hline Muhammad Yusuf & University of Trunojoyo, Madura & Indonesia \\
\hline Sri Zuliana & UIN Sunan Kalijaga & Indonesia \\
\hline Nur Zareen Zulkarnain & Universiti Teknikal Malaysia Melaka & Malaysia \\
\hline
\end{tabular}




\section{Additional Reviewers}

\begin{tabular}{|c|c|c|}
\hline Intan Ermahani A. Jalil & Universiti Teknikal Malaysia Melaka & Malaysia \\
\hline Mohd Helmy Abd Wahab & Universiti Tun Hussein Onn Malaysia & Malaysia \\
\hline MS. Hendriyawan Achmad & Yogyakarta University of Technology & Indonesia \\
\hline I Wayan Adiyasa & Gadjah Mada University & Indonesia \\
\hline Ali Qusay Al-Faris & Assumption College & USA \\
\hline Shajith Ali & SSN College of Engineering, Chennai & India \\
\hline Gede Angga Pradiptha & Institut Teknologi dan Bisnis STIKOM Bali & Indonesia \\
\hline Dhani Ariatmanto & Universitas Amikom Yogyakarta & Indonesia \\
\hline Agus Sasmito Aribowo & Universitas Pembangunan Nasional Veteran Yogyakarta & Indonesia \\
\hline Andria Arisal & Indonesian Institute of Sciences & Indonesia \\
\hline Arti Arya & PESIT-Bangalore South Campus & India \\
\hline Aslina Baharum & Universiti Malaysia Sabah & Malaysia \\
\hline Maria Chiara Caschera & CNR & Italy \\
\hline Wichian Chutimaskul & King Mongkut's University of Technology Thonburi & Thailand \\
\hline Domenico Ciuonzo & University of Naples Federico II, IT & Italy \\
\hline Ni Ketut Dewi Ari Jayanti & Institute of Technology and Business STIKOM Bali & Indonesia \\
\hline Andi Wahju Rahardjo Emanuel & Universitas Atma Jaya Yogyakarta & Indonesia \\
\hline Ahmad Nurul Fajar & Bina Nusantara University & Indonesia \\
\hline Anggit Ferdita Nugraha & Universitas AMIKOM Yogyakarta & Indonesia \\
\hline Alireza Ghasempour & ICT Faculty & USA \\
\hline Seng Hansun & Universitas Multimedia Nusantara & Indonesia \\
\hline Hartatik Hartatik & STMIK AMIKOM Yogyakarta & Indonesia \\
\hline Mardhiya Hayaty & Universitas AMIKOM Yogyakarta & Indonesia \\
\hline Roberto Carlos Herrera Lara & National Polytechnic School & Ecuador \\
\hline Tonny Hidayat & Universitas AMIKOM Yogyakarta & Indonesia \\
\hline Danial Hooshyar & Korea University & Korea (South) \\
\hline Hozairi Hozairi & Islamic University of Madura & Indonesia \\
\hline Rini Indrayani & Universitas Amikom Yogyakarta & Indonesia \\
\hline Nurulisma Ismail & Universiti Malaysia Perlis & Malaysia \\
\hline Ramkumar Jaganathan & VLB Janakiammal College of Arts and Science & India \\
\hline Biao Jiang & The City University of New York & USA \\
\hline Sandy Kosasi & STMIK Pontianak & Indonesia \\
\hline Krisnawati Krisnawati & University of AMIKOM Yogyakarta & Indonesia \\
\hline Ryan Putranda Kristianto & Universitas Amikom Yogyakarta & Indonesia \\
\hline Sumit Kushwaha & KNIT Sultanpur & India \\
\hline Pavel Loskot & Swansea University & United Kingdom (Great Britain) \\
\hline Prita Dewi Mariyam & Universitas Indonesia & Indonesia \\
\hline Ratheesh Kumar Meleppat & University of California Davis & USA \\
\hline Amrit Mukherjee & Jiangsu University & China \\
\hline Syibrah Naim & Universiti Sains Malaysia & Malaysia \\
\hline Asro Nasiri & University of Amikom Yogyakarta & Indonesia \\
\hline Shah Nazir & University of Peshawar & Pakistan \\
\hline Ruzelita Ngadiran & Universiti Malaysia Perlis & Malaysia \\
\hline Hanung Adi Nugroho & Universitas Gadjah Mada & Indonesia \\
\hline Prapto Nugroho & Universitas Gadjah Mada & Indonesia \\
\hline Ilker Ali Ozkan & Selcuk University & Turkey \\
\hline Oktavia Ayu Permata & Institut Teknologi Telkom Surabaya & Indonesia \\
\hline Gede Pramudya Ananta & Universiti Teknikal Malaysia Melaka & Malaysia \\
\hline Prihandoko Prihandoko & University of Gunadarma & Indonesia \\
\hline Guntur Dharma Putra & University of New South Wales & Australia \\
\hline Lesnanto Multa Putranto & UGM & Indonesia \\
\hline Suwanto Raharjo & Informatics of The Institut Sains \& Teknologi AKPRIND Yogyakarta & Indonesia \\
\hline Bambang Riyono & Gadjah Mada University & Indonesia \\
\hline Leo Santoso & Petra Christian University & Indonesia \\
\hline Vaibhav Dudhaji Saundarmal & Marathwada Institute of Technology, Aurangabad & India \\
\hline Enny Sela & Universitas Teknologi Yogyakarta & Indonesia \\
\hline Ririn Septrisulviani & Universitas Gadjah Mada & Indonesia \\
\hline Amel Serrat & USTO MB & Algeria \\
\hline
\end{tabular}




\begin{tabular}{|l|l|l|}
\hline Lilis Susanti Setianingsih & Universitas Gadjah Mada & Indonesia \\
\hline Noor Akhmad Setiawan & Universitas Gadjah Mada & Indonesia \\
\hline Iwan Setyawan & Satya Wacana Christian University & Indonesia \\
\hline Abdul Samad Shibghatullah & UCSI University & Malaysia \\
\hline Achmad Solichin & Universitas Budi Luhur & Indonesia \\
\hline lickho Song & Korea Advanced Institute of Science and Technology & Korea (South) \\
\hline Andi Sunyoto & Universitas AMIKOM Yogyakarta & Indonesia \\
\hline Melwin Syafrizal & Universitas AMIKOM Yogyakarta & Indonesia \\
\hline Srinivasulu Tadisetty & Kakatiya University College of Engineering and Technology & India \\
\hline Chakib Taybi & Mohammed First University & Morocco \\
\hline Ivanna Timotius & Satya Wacana Christian University & Indonesia \\
\hline Evi Triandini & Institut Teknologi dan Bisnis STIKOM Bali & Indonesia \\
\hline Anik Vega Vitianingsih & Universitas Dr Soetomo & Indonesia \\
\hline Leong Wen Chek & University of Malaya & Malaysia \\
\hline Adi Wibowo & Diponegoro University & Indonesia \\
\hline Ainul Yaqin & Universitas Amikom Yogyakarta & Indonesia \\
\hline Thaweesak Yingthawornsuk & King Mongkut's University of Technology Thonburi & Thailand \\
\hline Uky Yudatama & Universitas Indonesia & Indonesia \\
\hline Sri Utami Zuliana & UIN Sunan Kalijaga & Indonesia \\
\hline
\end{tabular}




\section{4th International Conference on Information Technology, Information Systems and Electrical Engineering (ICITISEE)}

Parallel Session 1-A

Evaluation of Golomb Ruler Optimum Performance for NG-PON2 Networks

Satrio Priambodo (Telkom University, Indonesia), Brian Pamukti (Telkom University, Indonesia), Akhmad Hambali (Telkom University, Indonesia)

Entropy and Information Gain Analysis on Low Cost BCI for Motorbike Users to Prevent Accident

Rolly Maulana Awangga (Institut Teknologi Bandung \& Politeknik Pos Indonesia, Indonesia), Syafrial Fachri Pane (Applied Bachelor Program of Informatics Engineering, Politeknik Pos Indonesia, Indonesia), Dinda Majesty (Politeknik Pos Indonesia, Indonesia), Moch Yusuf Asyhari (Universitas Islam Indonesia, Indonesia)

Study on C-Band Electromagnetic Wave Absorber made of S-Ring Resonator

Farhan Fathir Lanang (Telkom University, Indonesia), Levy Olivia Nur (Telkom University, Indonesia), Budi Syihabuddin (Telkom University, Indonesia), Bambang Setia Nugroho (Telkom University, Indonesia), Agus D. Prasetyo (Telkom University, Indonesia), Heroe Wijanto (Telkom University, Indonesia)

Performance evaluation of M-ary modulated DCO-OFDM in an Indoor Visible Light Communication System

Nurul Fatma Milia (Telkom University, Indonesia), Erna Sugesti (Telkom University, Indonesia), Desti Madya Saputri (Telkom University, Indonesia), Brian Pamukti (Telkom University, Indonesia)

Impact Analysis of Location and Penetration Level of DFIG on Small Signal Stability of Power System

Avrin Nur Widiastuti (UGM, Indonesia), Sarjiya Sarjiya (Gadjah Mada University, Indonesia), Sasongko Hadi (Universitas Gadjah Mada, Indonesia)

Analysis of Transient Signal using Hilbert-Huang Transform for Chatter Monitoring in Turning Process

Agus Susanto (State Polytechnic of Madiun, Indonesia), Keiji Yamada (Hiroshima University, Japan), Ryutaro Tanaka (Hiroshima University, Japan), Muizuddin Azka (Agency for the Assessment and Application of Technology (BPPT), Indonesia), Katsuhiko Sekiya (Hiroshima University, Japan), Murman Dwi Prasetio (Telkom University, Indonesia), Putri Novia (State Polytechnic of Madiun, Indonesia)

Parallel Session 1-B

Automatic Cacao Pod Detection Under Outdoor Condition Using Computer Vision

Yulia Ekawaty (Universitas Hasanuddin, Indonesia), Indrabayu A (Hasanuddin University, Indonesia), Intan Sari Areni (Hasanuddin University, Indonesia)

Using Big Data and Al to Examine Product Engagement in Social Media Influencer Posts

Stuart Barnes (King's College London, United Kingdom (Great Britain)), Richard Rutter (Australian College of Kuwait, United Kingdom (Great Britain))

The Best Parameters to Select Instagram Account for Endorsement using Web Scraping

Muhammad Ichwandar Akrianto (Universitas Amikom Yogyakarta, Indonesia), Anggit Dwi Hartanto (Universitas Amikom Yogyakarta,

Indonesia), Adri Priadana (Universitas Jenderal Achmad Yani Yogyakarta, Indonesia)

Decision Support System for Boarding house Search Using Topsis Algorithm

Ainul Yaqin (Universitas Amikom Yogyakarta, Indonesia), Akhmad Dahlan (Universitas Amikom Yogyakarta, Indonesia), Tonny Hidayat (Universitas AMIKOM Yogyakarta, Indonesia), Reza Mardiansyah Putra (Amikom, Indonesia)

The Selection of Periodic Salary Increment of Civil Servants using Fuzzy MADM

Wahyuni Eka Sari (Politeknik Pertanian Negeri Samarinda, Indonesia), Silmi Fauziati (Universitas Gadjah Mada, Indonesia)

Time-Frequency Analysis (TFA) method for load identification on Non-Intrusive Load Monitoring

Nur Iksan (Universitas Negeri Semarang, Indonesia), Erika Devi Udayanti (Universitas Dian Nuswantoro, Indonesia)

Parallel Session 1-C

Automatically Regulates Non Player Character Behavior Using Fuzzy Logic As An Artificial Intelligence Mechanism For Action Makers Tonny Hidayat (Universitas AMIKOM Yogyakarta, Indonesia), Ika Astuti (Universitas Amikom Yogyakarta, Indonesia), Akhmad Dahlan (Universitas Amikom Yogyakarta, Indonesia)

Comparison Analysis of the Implementation of the AHP and AHP-PROMETHEE Methods for the Selection of Trainees

Dewi Anisa Istiqomah (Universitas AMIKOM Yogyakarta, Indonesia), Vikky Aprelia (Universitas AMIKOM Yogyakarta, Indonesia)

Clustering K Means for Criteria Weighting With Improvement Result of Alternative Decisions Using SAW and TOPSIS

Erna Daniati (Indonesia \& Universitas Nusantara PGRI Kediri, Indonesia), Hastari Utama (Universitas Amikom Yogyakarta, Indonesia)

Animal metamorphosis learning media using android- Based augmented reality technology

Agus Purwanto (Lecturer of University Amikom Yogyakarta \& CREATIVE DIRECTOR OF PARAMA CREATIVE, Indonesia), Mei Parwanto Kurniawan (University of AMIKOM Yogyakarta, Indonesia), Ahmad Zaid Rahman (Universitas AMIKOM Yogyakarta, Indonesia)

Augmented Reality of Android-Based Learning Media of Sun and Earth Structure

Mei Parwanto Kurniawan (University of AMIKOM Yogyakarta, Indonesia), Agus Purwanto (Lecturer of University Amikom Yogyakarta \& CREATIVE DIRECTOR OF PARAMA CREATIVE, Indonesia), Muhammad Fahmi Mansur (University of Amikom Yogyakarta, Indonesia) 
Implementation Least Means Square Algorithm for Real-Time Active Noise Cancellation on FPGA

Muhammad Nur Shahreen Osman (Universiti Teknologi MARA, Malaysia), Ili Shairah Abdul Halim (Universiti Teknologi MARA \& Faculty of

Electrical Engineering, Malaysia), Siti Lailatul Mohd Hassan (Universiti Teknologi Mara, Malaysia), A'zraa Afhzan Ab Rahim (Universiti

Teknologi MARA, Malaysia), Noor Ezan Abdullah (Universiti Teknologi Mara, Malaysia)

\section{Parallel Session 1-D}

Comparison of Pornographic Image Classification based on Texture, Color, and Shape Features

I Wayan Pandu Swardiana (Universitas AMIKOM Yogyakarta, Indonesia), Arief Setyanto (Universitas AMIKOM Yogyakarta, Indonesia), Sudarmawan Sudarmawan (AMIKOM Yogyakarta University, Indonesia)

Implementation of 2DPCA and SOM Algorithms to Determine Sex According to Lip Shapes

Nor Hikmah (Universitas AMIKOM, Indonesia)

Comparison of Scale Invariant Feature Transform and Speed Up Robust Feature for Image Forgery Detection Copy Move

Reflan Nuari (Amikom University Yogyakarta, Indonesia), Ema Utami (Universitas Amikom Yogyakarta, Indonesia), Suwanto Raharjo (Informatics of The Institut Sains \& Teknologi AKPRIND Yogyakarta, Indonesia)

Potential Detection of Lentigo Maligna Melanoma on Solar Lentigines Image Based on Android

Casi Setianingsih (Telkom University, Indonesia)

Personality Features Identification from Handwriting Using Convolutional Neural Networks

Sri Fatimah (Universitas Jenderal Achmad Yani, Indonesia), Esmeralda Contessa Djamal (Universitas Jenderal Achmad Yani, Indonesia),

Faiza Renaldi (Universitas Jenderal Achmad Yani, Indonesia)

Comparison of Naive Bayes and K-NN method on Tuition Fee Payment Overdue Prediction

Kusrini Kusrini (AMIKOM Yogyakarta University, Indonesia), Emha Taufiq Luthfi (Universitas AMIKOM Yogyakarta, Indonesia), Muqorobin

Muqorobin (Universitas AMIKOM Yogyakarta, Indonesia), Robi Abdullah (Universitas AMIKOM Yogyakarta, Indonesia)

\section{Parallel Session 1-E}

Impact of Device Orientation for Visible Light Communication in Closed Room

Amirullah Wijayanto (Telkom University, Indonesia), Kris Sujatmoko (Telkom University, Indonesia), Brian Pamukti (Telkom University, Indonesia)

Evaluate Number of LED on Reflector Room for Optical Wireless Communication

Dyndra Ramadhanti (Telkom University, Indonesia), Brian Pamukti (Telkom University, Indonesia), Kris Sujatmoko (Telkom University, Indonesia)

Blockchain-based Secure Data Storage for Door Lock System

Ulfah Nadiya (Indonesian Institute of Science, Indonesia), Muhammad Ilham Rizqyawan (Indonesian Institute of Sciences, Indonesia), Oka Mahendra (Indonesian Institute of Sciences, Indonesia)

Detection System for Cigarette Smoke

Junaidy B Sanger (Universitas Katolik De La Salle Manado, Indonesia), Lanny Sitanayah (Universitas Katolik De La Salle Manado, Indonesia), Vivie D. Kumenap (Universitas Katolik De La Salle Manado, Indonesia)

A Novel Approach to Resource Starvation Attacks on MQTT Brokers

Ricardo Da Paz (Edith Cowan University, Australia), Aiden Sehovic (Edith Cowan University, Australia), David Cook (Edith Cowan University, Australia), Leisa J Armstrong (Edith Cowan University, Australia)

Design and Implementation of Node Gateway with MQTT and CoAP Protocol for IoT Applications

Ahmad Zainudin (Politeknik Elektronika Negeri Surabaya, Indonesia), Mohamad Fahmi Syaifudin (PENS, Indonesia), Nanang Syahroni (Politeknik Elektronika Negeri Surabaya, Indonesia)

Parallel Session 2-A

Electroencephalograph Recording with Ten-Twenty Electrode System Based on Arduino Mega 2560 Titis Bagus Kurnianadi (Soegijapranata Catholic University, Indonesia), Florentinus Budi Setiawan (Soegijapranata Catholic University, Indonesia)

Diminish the Peak Value of the Cogging Torque by Modifying of the Stator Teeth Tip Geometry

Herlina Wahab (Sriwijaya University \& University of Indonesia, Indonesia), Rudy Setiabudy (Universitas Indonesia (UI), Indonesia), Syamsuri Zaini (Sriwijaya University, Indonesia)

Level Crossing Rate Impact on Routing Performance in Adhoc Networks for Device-to-Device Communication

Istikmal Istikmal (Telkom University, Indonesia), Edwar Edwar (Telkom University, Indonesia)

An Adaptive Scaling Factor for Multiple Watermarking Scheme

Dhani Ariatmanto (Universitas Amikom Yogyakarta, Indonesia), Ferda Ernawan (Universiti Malaysia Pahang, Malaysia) 
Development of Internet-of-Things based Building Monitoring System for Supporting the Disaster Mitigation in The City

Asep Najmurrokhman (Universitas Jenderal Achmad Yani, Indonesia), Kusnandar Kusnandar, k (UNJANI \& UNJANI, Indonesia), Udin Komarudin (Universitas Jenderal Achmad Yani, Indonesia), Ahmad Daelami (Universitas Jenderal Achmad Yani, Indonesia), Restu Arisandy (Universitas Jenderal Achmad Yani, Indonesia)

MQTT Performance as a Message Protocol in an loT based Chili Crops Greenhouse Prototyping

Dania Eridani (Diponegoro University, Indonesia), Kurniawan Martono (Diponegoro University, Indonesia)

Development of Automatic Waste Segregator with Monitoring System

A'zraa Afhzan Ab Rahim (Universiti Teknologi MARA, Malaysia), Nurisha Hania Kamarudin (UiTM, Malaysia), Noor Ezan Abdullah (Universiti Teknologi Mara, Malaysia), Ili Shairah Abdul Halim (Universiti Teknologi MARA \& Faculty of Electrical Engineering, Malaysia), Siti Lailatul Mohd Hassan (Universiti Teknologi Mara, Malaysia)

Quantization Effect on 5 G Millimeter Wave Communication

Nasrullah Armi (Indonesian Institute of Sciences \& Telkom University, Indonesia), Chaeriah Bin Ali Wael (Indonesian Institute of Sciences, Indonesia), Arumjeni Mitayani (Indonesian Institute of Sciences, Indonesia), Arief Suryadi Satyawan (Waseda University, Japan \& Indonesian Institute of Sciences, Indonesia), Galih Nugraha Nurkahfi (Indonesian Institute of Science(LIPI), Indonesia)

\section{Parallel Session 2-B}

Human Perception Evaluation toward End of File Steganography Method's Implementation Using Multimedia File (Image, Audio, and Video) Rini Indrayani (Universitas Amikom Yogyakarta, Indonesia)

Hybrid Encryption Technique using Cyclic Bit Shift and RC4

Dina Evita Sari (Dian Nuswantoro University, Indonesia), Happy Niti Noor Muchsin (Dian Nuswantoro University, Indonesia), De Rosal Ignatius Moses Setiadi (Dian Nuswantoro University, Indonesia), Christy Atika Sari (Dian Nuswantoro University, Indonesia), Eko Hari Rachmawanto (Dian Nuswantoro University, Indonesia)

Steganographic-Algorithm and Length Estimation Classification on MP3 Steganalysis with Convolutional Neural Network Muhammad Rizki Duwinanto (Bandung Institute of Technology, Indonesia), Rinaldi Munir (Institut Teknologi Bandung, Indonesia)

QIM-based Audio Watermarking using Polar-based Singular Value in DCT Domain

Gelar Budiman (Telkom University \& Institute Technology Bandung, Indonesia), Ledya Novamizanti (Telkom University, Indonesia), Allwinnaldo Allwinnaldo (Telkom University, Indonesia)

Dual Encryption Method for File Security

Daniel Mahardika Yusuf (Dian Nuswantoro University, Indonesia), De Rosal Ignatius Moses Setiadi (Dian Nuswantoro University, Indonesia) Christy Atika Sari (Dian Nuswantoro University, Indonesia), Eko Hari Rachmawanto (Dian Nuswantoro University, Indonesia), Rabei Raad Al (Universiti Tun Hussein Onn Malaysia, Malaysia)

Design of Blockchain-Based Electronic Election System Using Hyperledger: Case of Indonesia

Donny Seftyanto (Badan Siber dan Sandi Negara, Indonesia), Amiruddin Amiruddin (Sekolah Tinggi Sandi Negara \& Badan Siber dan Sandi Negara, Indonesia), Arif Rahman Hakim (Sekolah Tinggi Sandi Negara, Indonesia)

Mitigation of Cryptojacking Attacks Using Taint Analysis

Arief Dwi Yulianto (Telkom University, Indonesia), Muhammad Al Makky (Telkom University, Indonesia), Parman Sukarno (Telkom University, Indonesia), Aulia Arif Wardana (Telkom University, Indonesia)

User Satisfaction Levels Sentiment Analysis Toward Goods Delivery Service On Twitter Using Support Vector Machine Algorithm (SVM)

Andia Enggar Mayasari (Universitas AMIKOM Yogyakarta, Indonesia), Anggit Dwi Hartanto (Universitas Amikom Yogyakarta, Indonesia)

Parallel Session 2-C

Planar Dipole MIMO Array Antenna for Mobile Robot Communications at $5.6 \mathrm{GHz}$

Muhsin Muhsin (Institut Teknologi Telkom Surabaya, Indonesia)

Natural Disaster Application on Big Data and Machine Learning: A Review

Rania Arinta (Universitas Atma Jaya Yogyakarta, Indonesia), Andi Wahju Rahardjo Emanuel (Universitas Atma Jaya Yogyakarta, Indonesia)

Machine Learning Classifiers for Autism Spectrum Disorder: Review

Dadang Eman (Universitas Atma Jaya Yogyakarta, Indonesia), Andi Wahju Rahardjo Emanuel (Universitas Atma Jaya Yogyakarta, Indonesia)

Comparison of Classification Methods using Historical Loan Application Data

Yohanes R. Laberto Kelen (Universitas Atma Jaya Yogyakarta, Indonesia), Andi Wahju Rahardjo Emanuel (Universitas Atma Jaya Yogyakarta, Indonesia)

The Undersampling Effects on RANDSHUFF Oversampling Algorithms

Tora Fahrudin (University of Telkom, Indonesia)

Meta-Algorithms for Improving Classification Performance in the Web-phishing Detection Process

Anggit Ferdita Nugraha (Universitas AMIKOM Yogyakarta, Indonesia), Luthfia Rahman (Universitas Gadjah Mada, Indonesia)

The Prototype of Decision Support System For Selecting The Lands of Crops

Dema Mathias Lumban Tobing (Universitas Amikom Yogyakarta, Indonesia), Julia Kurniasih (Amikom University, Indonesia), Yulius Nahak Tetik (Amikom University, Indonesia), Kusrini Kusrini (AMIKOM Yogyakarta University, Indonesia) 
Comparison Of SIFT and SURF Methods For Porn Image Detection

Hartatik Hartatik (STMIK AMIKOM Yogyakarta, Indonesia), Arief Setyanto (Universitas AMIKOM Yogyakarta, Indonesia), Kusrini Kusrini

(AMIKOM Yogyakarta University, Indonesia), I Made Artha Agastya (Universitas Amikom Yogyakarta, Indonesia)

Parallel Session 2-D

Self-Organizing Map (SOM) For Diagnosis Coronary Heart Disease

Triyanna Widiyaningtyas (Universitas Negeri Malang, Indonesia), Ilham A. E. Zaeni (State University of Malang, Indonesia), Putri Wahyuningrum (Universitas Negeri Malang, Indonesia)

Effect of Giving N Value on ADASYN-N Method for Classification of Imbalanced Nominal Data

Sri Rahayu (Universitas Janabadra, Indonesia), Jeffry Andhika Putra (Universitas Janabadra, Indonesia), Yumarlin MZ (Universitas Janabadra, Indonesia)

Sentiment Analysis in Airline Tweets Using Mutual Information for Feature Selection

Hastari Utama (Universitas Amikom Yogyakarta, Indonesia)

Optimization Of Parameter Support Vector Machine (SVM) using Genetic Algorithm to Review GO-JEK Services

Windha Mega Pradnya Dhuhita (Universitas AMIKOM Yogyakarta, Indonesia), Haryoko Haryoko (University of AMIKOM Yogyakarta,

Indonesia)

A Review of Long Short-Term Memory Method for Hate Speech Classification on Twitter

Syahrul Syafaat Syam (Telkom University, Indonesia), Budhi Irawan (Telkom University, Indonesia), Casi Setianingsih (Telkom University, Indonesia)

Comparison Performance of Decision Tree Classification Model for Spam Filtering with or without the Recursive Feature Elimination (RFE) Approach

Ahmad Fikri Zulfikar (University of Pamulang \& Universiry of Bina Nusantara, Indonesia)

Comparison of Modulation Schemes toward Coverage Area in indoor Visible Light Communication

Andrik Supadiyanto (Telkom University, Indonesia), Brian Pamukti (Telkom University, Indonesia), Desti Madya Saputri (Telkom University, Indonesia), Nur Andini (Telkom University, Indonesia)

Parallel Session 2-E

Speech recognition for Indonesian language and its application to home automation

Zulkarnaen Hatala (Politeknik Negeri Ambon, Indonesia)

Aspect and Opinion Word Extraction on Opinion Sentences in Bahasa Indonesia using Rule Based Generated from Regular Expression Yuliana Setiowati (Politeknik Elektronika Negeri Surabaya, Indonesia), Fitri Setyorini (Politeknik Elektronika Negeri Surabaya, Indonesia), Afrida Helen (Universitas Padjadjaran (Unpad), Indonesia)

The Use Matriks of Linear and Quadratic Regression to Predict Number Electricity Distributed in Indonesia

Desty Rakhmawati (Universitas Amikom Purwokerto, Indonesia), Hendra Marcos (STMIK AMIKOM Purwokerto, Indonesia), Utami Puspita (Universitas AMIKOM Purwokerto, Indonesia), Uswatun Hasanah (STMIK Amikom Purwokerto, Indonesia)

An Optimization of a Lexicon Based Sentiment Analysis Method on Indonesian App Review

Bayu Trisna Pratama (Universitas Amikom Yogyakarta, Indonesia), Ema Utami (Universitas Amikom Yogyakarta, Indonesia), Andi Sunyoto (Universitas AMIKOM Yogyakarta, Indonesia)

Classification of Citation Sentence for Filtering Scientific References

Ghoziyah Haitan Rachman (Institut Teknologi Bandung, Indonesia), Masayu Leylia Khodra (Institut Teknologi Bandung, Indonesia), Dwi H Widyantoro (Institut Teknologi Bandung, Indonesia)

Indonesian Part of Speech Tagging Using Hidden Markov Model - Ngram \& Viterbi

Denis Eka Cahyani (Universitas Sebelas Maret, Indonesia)

Mining Student Feedback to Improve the Quality of Higher Education through Multi Label Classification, Sentiment Analysis, and Trend Topic Calandra Alencia Haryani (Universitas Indonesia \& Universitas Pelita Harapan, Indonesia), Achmad Hidayanto (University of Indonesia, Indonesia), Nur Fitriah Ayuning Budi (Universitas Indonesia, Indonesia), Zaenal Abidin (Universitas Negeri Semarang, Indonesia), Theresia Wati (Universitas Pembangunan Nasional Veteran Jakarta, Indonesia)

Mobile Business Intelligence Assistant ( $m$-BELA) for Higher Education Executives

Mohamad Irwan Afandi (Universitas Pembangunan Nasional Veteran Jawa Timur, Indonesia), Eka Dyar Wahyuni (Universitas Pembangunan Nasional Veteran Jawa Timur, Indonesia), Siti Mukaromah (Universitas Pembangunan Nasional Veteran Jawa Timur, Indonesia)

Parallel Session 3-A

Design of PID Controllers for Speed Control of Three Phase Induction Motor Based on Direct-Axis Current (Id) Coordinate Using IFOC Indra Ferdiansyah (Politeknik Elektronika Negeri Surabaya, Indonesia), Diah Yanaratri (Politeknik Elektronika Negeri Surabaya, Indonesia), Lucky Pradigta Setiya Raharja (Politeknik Elektronika Negeri Surabaya, Indonesia), Era Purwanto (Electronic Engineering Polytechnic Institute Of Surabaya, Indonesia) 
Dynamic Source Routing and Optimized Link State Routing Performance in Multipath Fading Environment with Dynamic Network Topology Istikmal Istikmal (Telkom University, Indonesia), Agus Subekti (Indonesian Institute of Sciences, Indonesia), Doan Perdana (Telkom University, Indonesia), Ridha Negara (Telkom University, Indonesia), Arif Indra Irawan (Telkom University, Indonesia), Sussi Sussi (Telkom University, Indonesia)

Comparative Analysis of Codec G.729 and G.711 on IEEE 802.11AH with MCS and Raw Slot Change Mechanism for VOIP Service Doan Perdana (Telkom University, Indonesia), Istikmal Istikmal (Telkom University, Indonesia)

Flooding Detection System Based on Water Monitoring and ZigBee Mesh Protocol

Herman Yuliandoko (State Polytehnic of Banyuwangi, Indonesia), Abdul Rohman (State Polytechnic of Banyuwangi, Indonesia)

Performance Comparison of Blackman, Bartlett, Hanning, and Kaiser Window for Radar Digital Signal Processing

Sulis Tyaningsih (Indonesian Institute of Science (LIPI), Indonesia), Prasetyo Putranto (Indonesian Institute of Sciences, Indonesia),

Taufiqqurrachman Taufiq (National Taiwan University of Science and Technology \& Indonesian Institute of Sciences, Indonesia), Winy Desvasari (Indonesian Institute of Sciences, Indonesia), Yusuf Nur Wijayanto (Indonesian Institute of Sciences (LIPI), Indonesia),

Pamungkas Daud (Indonesian Institute of Sciences, Indonesia), Dadin Mahmudin (Indonesian Institute of Sciences (LIPI), Indonesia), Deni

Permana Kurniadi (LIPI, Indonesia), Arief Rahman (Indonesian Institute of Sciences, Indonesia), Sri Hardiati (Indonesian Institute of Science (LIPI), Indonesia), Arie Setiawan (Indonesian Institute of Sciences, Indonesia), Fajri Darwis (Indonesian Institute of Sciences (LIPI),

Indonesia), Eko Pristianto (Indonesian Institute of Sciences, Indonesia)

Implementation of Algorithm Rabin-Karp for Thematic Determination of Thesis

Ainul Yaqin (Universitas Amikom Yogyakarta, Indonesia), Akhmad Dahlan (Universitas Amikom Yogyakarta, Indonesia), Reno Diandika Hermawan (University of Amikom, Indonesia)

Hybrid Resampling for Imbalanced Class Handling on Web Phishing Classification Dataset

Yoga Pristyanto (Universitas Amikom Yogyakarta, Indonesia), Akhmad Dahlan (Universitas Amikom Yogyakarta, Indonesia)

Parallel Session 3-B

Modeling of Time Series Data Prediction using Fruit Fly Optimization Algorithm and Triple Exponential Smoothing

Ryan Putranda Kristianto (Universitas Amikom Yogyakarta, Indonesia)

A Novel Ant Colony Optimization Algorithm for Waste Collection Problem

Sarifah Putri Raflesia (Universitas Sriwijaya \& Institut Teknologi Bandung, Indonesia), Anugrah K Pamosoaji (Universitas Atma Jaya

Yogyakarta, Indonesia)

Fuzzy Hierarchical Model and Particle Swarm Optimization in Gas Leakage Detector Mobile Robot

Kanda Januar Miraswan (Universitas Sriwijaya, Indonesia), Muhammad Ali Buchari (Universitas Sriwijaya, Indonesia), Rizki Kurniati

(Universitas Sriwijaya, Indonesia)

Optimization of Weight Backpropagation with Particle Swarm Optimization for Student Dropout Prediction

Eka Yulia Sari (Universitas Amikom Yogyakarta, Indonesia), Kusrini Kusrini (AMIKOM Yogyakarta University, Indonesia), Andi Sunyoto (Universitas AMIKOM Yogyakarta, Indonesia)

The Mapping of Lighting Intensity from the Light Distribution on LED and CFL Lamps

Herlina Wahab (Sriwijaya University \& University of Indonesia, Indonesia), Rudy Setiabudy (Universitas Indonesia (UI), Indonesia)

Impact of STATCOM Installation on Power System's Voltage Stability Performance

Nurriza Kholifatulloh Hasanah (Universitas Gadjah Mada, Indonesia), Lesnanto Multa Putranto (UGM, Indonesia), Sasongko Hadi (Universitas Gadjah Mada, Indonesia), Febian Melwa Reksa Aditya (Universitas Gadjah Mada, Indonesia)

Analysis Of Overclock Ram Galax Hall Of Fame For Daily Needs

Rizqi Sukma Kharisma (Universitas AMIKOM Yogyakarta, Indonesia), Miko Kastomo Putro (Universitas AMIKOM Yogyakarta, Indonesia)

\section{Parallel Session 3-C}

Analysis of Review And Rating on Consumer Trust in Jakarta Taking Online Booking Queue Based on Tam Hendrico Andre (Bina Nusantara University, Indonesia), Sfenrianto Sfenrianto (Bina Nusantara University, Indonesia), Gunawan Wang (Bina Nusantara University, Indonesia), Pangondian Prederikus (Bina Nusantara, Indonesia)

Analysis of the Effect of Security and Trust on Buying Decision On the Tokopedia Mobile Apps

Edward Chandra (Bina Nusantara University, Indonesia), Stefanie Liu (Bina Nusantara University, Indonesia), Sfenrianto Sfenrianto (Bina Nusantara University, Indonesia), Gunawan Wang (Bina Nusantara University, Indonesia)

Behavior Intention of Information Technology Students in Using Youtube as Learning Resources

Paul Weniko (Bina Nusantara University, Indonesia), Gunawan Wang (Bina Nusantara University, Indonesia), Sfenrianto Sfenrianto (Bina Nusantara University, Indonesia), Muhammad Aldenny (Bina Nusantara University, Indonesia)

Analysis of the Effect of Trust on Purchase Intention in E-Commerce Integration for Vendors \& Event Organizer

Dina Ikramina (Bina Nusantara University, Indonesia), Sfenrianto Sfenrianto (Bina Nusantara University, Indonesia), Gunawan Wang (Bina Nusantara University, Indonesia)

Evaluating the Usability of Heuristics within Telegram using the Linear Regression Method

Rumini Rumini (Universitas AMIKOM Yogyakarta, Indonesia) 
Social Network Users Switching Platforms Behaviour: A Proposal for Research Explorations using a Mixed Method Approach Timothy McBush Hiele (Ming Chuan University, Taiwan), Andree E. Widjaja (Universitas Pelita Harapan, Indonesia), Calandra A. Haryani (Universitas Pelita Harapan, Tangerang, Indonesia)

Fingerprint Presence Fraud Detection Using Tight Clustering on Employee's Presence and Activity Data

Irfan Kamil (Institut Teknologi Bandung, Indonesia), Bambang Pharmasetiawan (Institut Teknologi Bandung, Indonesia)

\section{Parallel Session 3-D}

Analysis of Dominants Game Elements using the Sillaots Parameters and Octalysis Framework on the Google Play Store

Dema Mathias Lumban Tobing (Universitas Amikom Yogyakarta, Indonesia), Emma Utami (Universitas AMIKOM Yogyakarta, Indonesia), Hanif Fatta (Universitas AMIKOM Yogyakarta, Indonesia)

Pregnancy Mapping and Monitoring Web Based Geographic's Information System

Arief Munandar (Universitas AMIKOM Yogyakarta, Indonesia), Arief Setyanto (Universitas AMIKOM Yogyakarta, Indonesia), Suwanto Raharjo (Informatics of The Institut Sains \& Teknologi AKPRIND Yogyakarta, Indonesia), Gunawan Wicahyono (Amikom University Yogyakarta, Indonesia)

Integration of K-Means Clustering and Naive Bayes Classification Algorithms for Smart AC Monitoring and Control in WSAN

Ryan Putranda Kristianto (Universitas Amikom Yogyakarta, Indonesia), Banu Santoso (Universitas AMIKOM Yogyakarta, Indonesia)

Optimization of Transmission Expansion Planning Considering the System Losses: A Case Study of the Garver's 6-Bus System Afif Amalul Arifidin (Universitas Gadjah Mada, Indonesia), Sasongko Hadi (Universitas Gadjah Mada, Indonesia), Lesnanto Multa Putranto (UGM, Indonesia), Muhammad Yasirroni (Universitas Gadjah Mada, Indonesia)

Real-Time Irradiance Estimation Based on Maximum Power Current of Photovoltaic

Moh Syaiful Imam (Politeknik Elektronika Negeri Surabaya (PENS), Indonesia), Eka Prasetyono (Politeknik Elektronika Negeri Surabaya, Indonesia), Epyk Sunarno (Politeknik Elektronika Negeri Surabaya, Indonesia)

What are Customers Really Need in Ride Hailing Applications?Signaling Electronic Service Quality via E-CRM Features

Tifanny Nabarian (Universitas Indonesia, Indonesia, Indonesia), Yudho Sucahyo (University of Indonesia, Indonesia), Arfive Gandhi (Universitas Indonesia, Indonesia), Yova Ruldeviyani (Universitas Indonesia, Indonesia)

Realization of Point Cloud Maps Using ROS \& Visual Sensor on Raspberry Pi 3 Based Mobile Robot

Husnairi Ardan Miranto (Telkom University, Indonesia), Agung Nugroho Jati (Telkom University, Indonesia), Casi Setianingsih (Telkom University, Indonesia)

Parallel Session 3-E

Essential Blockchain Technology Adoption factors in Pharmaceutical Industry

Surjandy Surjandy (Bina Nusantara University, Indonesia), Erick Fernando (Bina Nusantara University, Indonesia), Meyliana Meyliana (Bina Nusantara University, Indonesia)

Performance Evaluation of XPath Routing Protocol in Data Center Network using NS3-Simulator

Gerry Wowiling (Universitas Indonesia, Indonesia), Hermawan Rahman Sholeh (University of Indonesia, Indonesia), Ruki Harwahyu (Universitas Indonesia \& Universitas Indonesia, Indonesia), Riri Fitri Sari (University of Indonesia, Indonesia)

Job Seeker Profile Classification of Twitter Data Using the Naïve Bayes Classifier Algorithm Based on the DISC Method

Anggit Dwi Hartanto (Universitas Amikom Yogyakarta, Indonesia), Ema Utami (Universitas Amikom Yogyakarta, Indonesia), Sumarni Adi (Universitas AMIKOM Yogyakarta, Indonesia), Harish Setyo Hudnanto (Universitas AMIKOM Yogyakarta, Indonesia)

The Impact Of Features Selection On Performance Of Artificial Neural Network In Diagnosis Of Diabetic Retinopathy

Tri Astuti (Universitas Amikom Purwokerto, Indonesia), Rizki Wahyudi (STMIK Amikom Purwokerto, Indonesia), Uswatun Hasanah (STMIK Amikom Purwokerto, Indonesia), Bambang Pilu Hartato (STMIK AMIKOM Purwokerto, Indonesia), Zanuar Rifa'i (Stmik Amikom Purwokerto, Indonesia)

RS Code and Compressive Sampling on Video Watermarking-based DWT-SVD

Ledya Novamizanti (Telkom University, Indonesia)

Trending Topic Classification for Single-Label Using Multinomial Naive Bayes (MNB) and Multi-Label Using K-Nearest Neighbors (KNN) Denis Eka Cahyani (Universitas Sebelas Maret, Indonesia)

Influence of Voltage System-Level to the dimensions and performance of Squirrel Cage Induction Motor Three Phase 50Hz $5 \mathrm{HP}$ for Electric Vehicles

Danang Wijaya (UGM, Indonesia), Iftitah Imawati (Universitas Gadjah Mada, Indonesia) 


\title{
Machine Learning Classifiers for Autism Spectrum Disorder: Review
}

\author{
$1^{\text {st }}$ Dadang Eman \\ Magister Teknik Informatika \\ Universitas Atma Jaya Yogyakarta \\ Yogyakarta, Indonesia \\ emanboy17@gmail.com \\ $2^{\text {nd }}$ Andi W.R Emanuel \\ Magister Teknik Informatika \\ Universitas Atma Jaya Yogyakarta \\ line 4: Yogyakarta, Indonesia \\ andi.emanuel@uajy.ac.id
}

\begin{abstract}
Autism Spectrum Disorder (ASD) is a brain development disorder that affects the ability to communicate and interact socially. There have been many studies using machine learning methods to classify autism including support vector machines, decision trees, naïve Bayes, random forests, logistic regression, K-nearest Neighbors and others. In this study provides a review on autism spectrum disorder by using a machine learning algorithm that is supervised learning. The initial study of the article was collected from a website provided articles were in according with this study, after going through the process of selecting articles $\mathbf{1 1}$ articles were eligible in this study. Based on the results obtained, that the most widely used algorithm in the literature study in this study is support vector machine (SVM) of $63.63 \%$, with the application of machine learning in the case of ASD expected to be able to accelerate and improve accuracy in determining a diagnosis.
\end{abstract}

Keywords - Autism Spectrum Disorder; Machine Learning, Classification.

\section{INTRODUCTION}

ASD is a condition of neurological development that affects the development of communication, behavior, social, with early detection and evidence that the development of interventions, in the beginning, has reduced the cost of treatment and building skills that people with autism spectrum disorder can be more independent later on [1]. Autism spectrum disorder is diagnosed in medical treatment by specialists who are competent in that field, in determining the diagnosis of autism spectrum disorder it still uses screening instruments that it requires and requires direct observation by clinical specialists [2]. Currently, the standard is still manual is used in assessing autism spectrum disorder by using standard assessment (ADOS) [3] and (ADI-R) [4], the procedure takes a long time and is relatively expensive to diagnose [2].

The importance of improving accurate methods to detect risks are faster than the current standard of care, Technology that has developed in the field of computers such as the internet of things, artificial intelligence, mobile applications, and machine learning helps human work to accelerate a process. One study that examined the instrument for assessing clinically autism spectrum disorder, in this study the instrument used was an observation autism diagnostic schedule (ADOS) using learning techniques [5].

In addition to assessing autism diagnostic observation schedule (ADOS) instruments, machine learning also assessed the autism diagnostic interview-revised (ADI-R) instrument to improve autism spectrum disorder screening [4] in this study using a support vector machine (SVM), data obtained from revised diagnostic interview autism and social responsiveness scale (SRS) scores.

Machine learning is able to detect a disease such as diagnosing someone who has autism, with machine learning increasing the accuracy and time of diagnosis to speed up the process of making decisions in dealing with patients with autism spectrum disorder (ASD) as early as possible so that patients get faster treatment so that patients are more independent in the future [6]. In this study provides a comprehensive review of research using machine learning, namely in autism spectrum disorder (ASD).

\section{LITERATURE REVIEW}

Advances in computing have been widely used in various Sciences disciplined one of them to know the developmental abnormalities of the nervous system that is autism. These abnormalities can be known since early childhood by making use of technologies that are already developing such as machine learning. Some research to predict the ASD using machine learning with a variety of methods including using Blood-based Gene Expression Signatures [7], behavioral distinction [8], metabolites in the FOCM/TS (folate-dependent one-carbon metabolism (FOCM)/transsulfuration (TS) [9], and home videos [10] [11].

Tariq et al. Conducted a study on the performance and accuracy of videos with machine learning in children. This study is useful for children who experience asd in developing countries that are limited to clinical resources so that they can establish diagnoses faster [10]. And another study, nd other research, using machine learning with home video can detect it ASD [11]. Research conducted by the Maenner et al, a machine learning approach to classify ADDM (Autism and Developmental Disabilities Monitoring) using words and phrases in children's evaluations using [12]. Identify the diagnosis of a child's ASD using an assistant robot [13] A robot that is used to identify the child's diagnosis of ASD for kindergarten based on children's response at the time of the call, in functional imitation (assess whether the child can imitate simple actions or not), joint attention, Play request. Other research also on imitation of movement [14], This study investigated whether the method machine 
learning-based data can be used to address some fundamental issues concerning parameters kinematic to classify between ASC (Autism spectrum condition) and controls neurotypical. The participants are kindly requested to observe the then mimics the order of hand movements, which contains two movements. Accuracy of five classification models (naïve Bayes, SVM RBF, SVM linear, decision tree, random forest) but the highest accuracy is SVM Classification and comparison of classification accuracy resulted from $86.7 \%$ use SVM classifier. Based on research $\mathrm{Bi}$ et al multiple support Vector Machine (SVM) for classifies the autism spectrum disorder (ASD) has an Accuracy of $96.15 \%$ of random SVM cluster [15].

Other studies, the SVM is also higher accuracy compared with another classification model [16], This study evaluates and compares the performance of four classifications machine learning. The number of samples used on the 1500 level 1 to ensure the purity of the object. 1000 data for training and testing data for 500 and 8 data sets obtained a sample of training each is $25,50,75,100,125$, 150, 175 and 200. Results: SVM is $92.6 \%$ regardless of the sample size, the overall accuracy of the SVM has much higher than NB, when relatively small training sample size. This study focuses on a literature study that discusses machine learning used in cases of autism spectrum disorder (ASD) using machine learning. In this study, only taking one of the techniques of application of machine learning is supervised learning. Researchers hope to be able to help other researchers in the domain of this research and can provide benefits from the techniques described in this study.

\section{METHOD AND MATERIAL}

The research method used to searching related research articles, using several websites that publish research papers are (1) ScienceDirect, (2) Google Scholar, (3) Springer, (4) PubMed, (5) ResearchGate In searching for research articles, the keywords used are autism spectrum disorder, autism, machine learning, supervised learning. To find research articles, researchers only take open source papers or articles on each website. Then the criteria for selecting research articles are based on the following inclusion criteria:

1. Research articles published in the last five years (2015-2019).

2. Research articles must be written in English

While the exclusion criteria are used to edit irrelevant articles as follows:

1. The research article does not discuss autism spectrum disorder, autism, machine learning and supervised learning.

2. Research articles that include subjects or tutorials After finding the research article, the researcher makes a summary of the article and then classifies the research articles and connects with each other. This study focuses on the use of machine learning in the scope of autism spectrum disorder (ASD). Search results include many articles that have no focus on machine learning in autism spectrum disorder (ASD) but use machine learning for other purposes. These articles were excluded from this study. Article search begins with the search process based on keywords. All related research articles are collected by reviewing titles and abstracts. Research articles were selected by including inclusion and exclusion criteria. This study also issued articles that were not published in English. In short, the focus is not to miss relevant documents in accordance with this study. Flow chart to identify eligible articles for this study but most were omitted by inclusion and exclusion criteria.
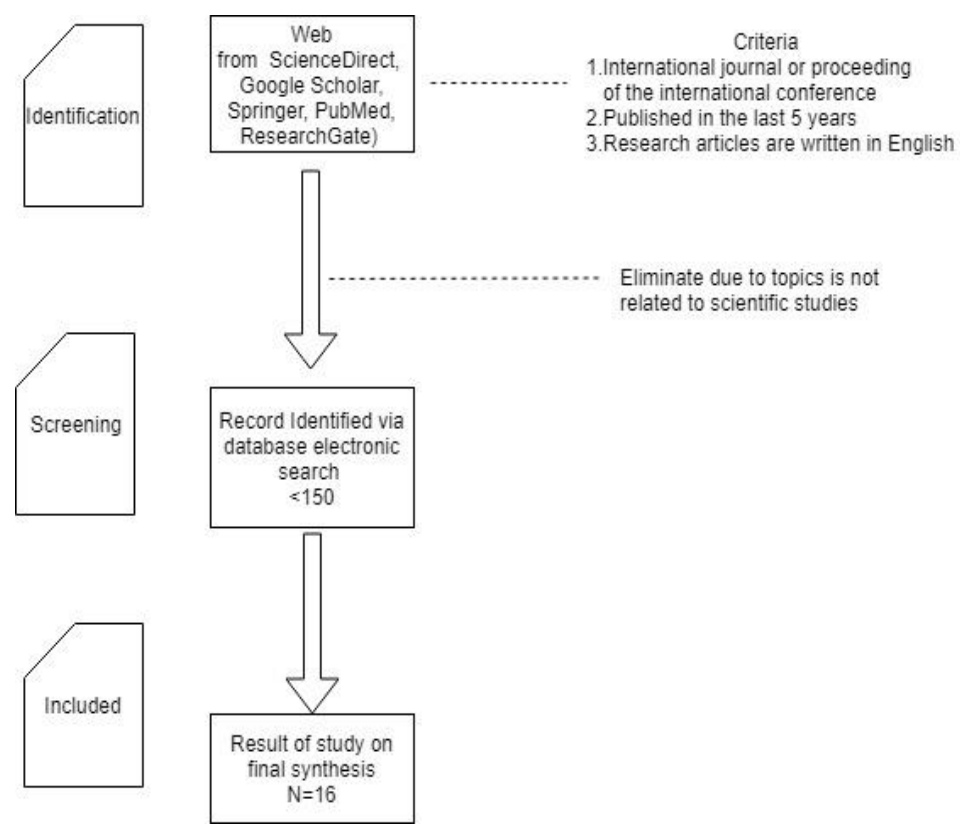

Fig 1. Flowchart Articles Identified

Figure 1. Steps for selecting articles are eligible for research. In this study, there were 16 journals that were in accord with the research criteria issued articles were not published in English. In short, the focus is not to miss relevant documents in accordance with this study.

\section{RESULT}

After selecting articles, match the needs of this study, we obtained 16 articles that discussed autism spectrum disorder (ASD) using machine learning with guided learning. Table 1 is the result of the article discussed in this study. Can be seen in table 1 below. Table 1 is the result of autism spectrum disorder article using supervised learning, in this study the supervised learning algorithm is used to detect autism in order to detect someone who has autism earlier, thus increasing the accuracy and time in diagnosing so as to speed up the decision making the process [6]. The research reviews this review literature is widely applied to supervised machine learning, which shows a superior classification model of each case or different dataset. Of the 16 research articles included, several articles that show the classification model used in Autism Spectrum Disorder (ASD), such as the naïve Bayes algorithm used in three research studies, uses decision tree algorithms used in four research studies, supporting vector machines used seven in the study, random forest was used five in the study, logistic regression was used in three studies, general linear models and KNN were used one in the research study respectively. Research articles that have been collected 
are grouped based on the model used in the study. There are several types of models used in table 2 .

Table 2. Machine Learning Methods Used

\begin{tabular}{|l|l|}
\hline Model & Citation \\
\hline Decision tree & {$[8][14][17][18][19]$} \\
\hline Random Forest & {$[5][14][12][20][8]$} \\
\hline SVM & {$[8][7][21][22][14][23]$} \\
& {$[5][24][25][26][19]$} \\
\hline Naïve Bayes Linear & {$[18][5][14]$} \\
\hline $\begin{array}{l}\text { Generalized } \\
\text { Model }\end{array}$ & {$[18]$} \\
\hline Logistic Regression & {$[5][8][18][27]$} \\
\hline KNN & {$[7]$} \\
\hline
\end{tabular}

Based on table 2, there are several types of algorithms used. For the type of support vector machine, the algorithm is most widely used because this algorithm is generally used to classify differences in groups of datasets are nonlinear. The Support vector machine (SVM) algorithm has been used in various studies including in this research review to classify someone to identify Autism Spectrum Disorder (ASD) the process of developing or increasing the accuracy of diagnosing autism spectrum disorder accelerates the various data obtained from kinematic data [14], blood gene [7], standard assessment [8] [21] [23] [5], face processing abnormality [22].

From these data can help distinguish patients who have Autism Spectrum Disorder (ASD) disorders or not who have Autism Spectrum Disorder (ASD). Based on research conducted by Li et al, by using kinematic data obtained from 16 respondents who experienced Autism Spectrum Disorder (ASD) and 14 respondents with a typically developing (TD), participants or respondents followed hand movements. From the results of observations, get kinematic parameters, which are as many 20 parameters are tested using the naïve Bayes algorithm that 20 parameters get 8 parameters. Then four logarithms used in this study were tested namely Support Vector Machine (SVM RBF and Linear), Random Forest, Naïve Bayes, Decision Tree, obtained from SVM and Naïve Bayes algorithm superior to the other two algorithms, Random Forest and Decision Tree. The highest value is Support Vector Machine (SVM) Linear with sensitivity $85.7 \%$, specificity $87.5 \%$ and accuracy $86.7 \%$ [14].

Other studies using facial scanning patterns conducted by Liu et $a l$, in this study using data sets obtained from eye movements on face recognition in children with SVM results obtained sensitivity $93.10 \%, 86.21 \%$ specificity and accuracy $88.51 \%$ [22]. In identifying the Autism Spectrum Disorder (ASD) facial scanning patterns were not only used but also used a blood-based gene to identify ASD, a study conducted by Oh et al, in this study using microarray data obtained from gene expression databases with 21 participant omnibus young adults. By using three algorithms namely SVM, KNN and LDA which were tested in this study. SVM and KNN are far superior to the LDA algorithm with each SVM and KNN getting the same overall value, namely sensitivity $100 \%$, specificity $87.5 \%$ and accuracy $93.8 \%$ [7].

The standard assessment used in identifying Autism Spectrum Disaster at the time of observing the potential behavior of errors in setting the diagnosis that machine learning is needed to increase accuracy in establishing the diagnosis of autism spectrum disorder (ASD), based on research conducted by Levy et al. using standard assessment, is the Autism Diagnostic Observation Schedule (ADOS) on module 2 and module 3 with respondents as many as 1319 with ASD and 70 with control on module 2 while in module 3 as many as 2870 with ASD and 273 controls with SVM linear L1 and logistic regression L2 for module $2(0.93)$ area under ROC curve and module 3 (0.95) area under ROC curve [23].

A similar study using ADOS (module 2 and module 3) was carried out by Kosmicki et al, in this study the total number of behaviors obtained from module 2 was 9 out of 28 behaviors, while in module 3 there were 12 of 28 behaviors from ASD with accuracy of $98.27 \%$ and $97.66 \%$ respectively to detect risks from ASD [5].

Another module to identify ASD is Autism Diagnostic Interview-Revised (ADI-R), Social Responsiveness Scale (SRS) [21] and social responsiveness scale score [8]. Research conducted by Bone et al, used the SVM algorithm to differentiate ASD and nonASD in participants as many 1264 with ASD and 462 Non ASD verbal individuals with results obtained sensitivity $89.2 \%$, specificity $59.0 \%$ for under 10 years of age while for ages above 10 years, sensitivity was $86.7 \%$ and specificity was $53.4 \%$, it was more efficient in increasing the accuracy of diagnosis [21]. While the research conducted by Duda et al, which uses social responsiveness scale (SRS) with 2925 samples (2775 with ASD and 150 with ADHD) use 6 algorithms including decision tree, random forest, LDA, category lasso, support vector classification and logistic regression in getting results four of the six algorithms had area under curve (AUC) 0.96 (LDA 0.964, categorical lasso 0.962 , logistic regression 0.962 and SVC 0.965) to distinguish between ASD and ADHD [8].

\section{CONCLUSION}

The literature review in this study observed autism spectrum disorder by using machine learning, which is supervised learning. Preliminary studies of articles were collected from online databases after going through the article selection process obtained by 16 research articles met the requirements in this study. Based on the results, it can be concluded that the most commonly used algorithm in this study is $63.63 \%$ support vector machine, $45.45 \%$ random forest, $36.36 \%$ decision tree. This study, although limited to literature study findings, has shown the potential of machine learning in the field of ASD, with the existence of machine learning applied to autism spectrum disorder (ASD) expected to be able to detect early can speed up the process of establishing and improving diagnostic accuracy compared to the still manual that early detection of future ASD sufferers can be more independent and the ability of machine learning in processing data can help further researchers who will conduct research on autism spectrum disorder (ASD). 


\section{REFERENCES}

P. O. Towle and P. A. Patrick, "Autism Spectrum Disorder Screening Instruments for Very Young Children: A Systematic Review," Autism Res. Treat., vol. 2016, pp. 1-29, 2016.

B. B. Sizoo et al., "Predictive validity of self-report questionnaires in the assessment of autism spectrum disorders in adults," Autism, vol. 19, no. 7, pp. 842-849, 2015.

T. Hirota, R. So, Y. S. Kim, B. Leventhal, and R. A. Epstein, “A systematic review of screening tools in non-young children and adults for autism spectrum disorder," Res. Dev. Disabil., vol. 80, no. May, pp. 1-12, 2018.

D. Bone, S. Bishop, M. P. Black, M. S. Goodwin, C. Lord, and S S. Narayanan, "Use of machine learning to improve autism screening and diagnostic instruments: effectiveness, efficiency, and multi-Instrument Fusion,” vol. 57, no. 8, pp. 927-937, 2017.

J. A. Kosmicki, V. Sochat, M. Duda, and D. P. Wall, "Searching for a minimal set of behaviors for autism detection through feature selection-based machine learning," Transl. Psychiatry, vol. 5, no. 2, pp. e514-7, 2015.

F. Thabtah, "Machine learning in autistic spectrum disorder behavioral research: A review and ways forward," Informatics Heal. Soc. Care, vol. 00, no. 00, pp. 1-20, 2018.

D. H. Oh, I. Bin Kim, S. H. Kim, and D. H. Ahn, "Predicting autism spectrum disorder using blood-based gene expression signatures and machine learning," Clin. Psychopharmacol. Neurosci., vol. 15, no. 1, pp. 47-52, 2017.

M. Duda, R. Ma, N. Haber, and D. P. Wall, "Use of machine learning for behavioral distinction of autism and ADHD," Transl. Psychiatry, vol. 6, no. November 2015, p. e732, 2016.

G. Li, O. Lee, and H. Rabitz, "High-efficiency classification of children with autism spectrum disorder," PLoS One, vol. 13, no. 2, pp. 1-23, 2018

Q. Tariq et al., "Detecting Developmental Delay and Autism Through Machine Learning Models Using Home Videos of Bangladeshi Children: Development and Validation Study," J. Med. Internet Res., vol. 21, no. 4, p. e13822, 2019.

[11] Q. Tariq, J. Daniels, J. N. Schwartz, P. Washington, H. Kalantarian, and D. P. Wall, "Mobile detection of autism through machine learning on home video: A development and prospective validation study," PLoS Med., vol. 15, no. 11, pp. 1-20, 2018.

[12] M. J. Maenner, M. Yeargin-Allsopp, K. N. Van Braun, D. L. Christensen, and L. A. Schieve, "Development of a machine learning algorithm for the surveillance of autism spectrum disorder," PLoS One, vol. 11, no. 12, pp. 1-11, 2016.

[13] F. Petrić et al., "Four tasks of a robot-assisted autism spectrum disorder diagnostic protocol: First clinical tests," Proc. 4th IEEE Glob. Humanit. Technol. Conf. GHTC 2014, pp. 510-517, 2014.

[14] B. Li, A. Sharma, J. Meng, S. Purushwalkam, and E. Gowen, "Applying machine learning to identify autistic adults using imitation: An exploratory study," PLoS One, vol. 12, no. 8, pp. 119, 2017.

[15] X. A. Bi, Y. Wang, Q. Shu, Q. Sun, and Q. Xu, "Classification of autism spectrum disorder using random support vector machine cluster," Front. Genet., vol. 9, no. FEB, pp. 1-10, 2018. learning classifiers for object-based land cover classification using very high resolution imagery," Remote Sens., vol. 7, no. 1, pp. 153-168, 2015.

[17] K. Hyde, A. J. Griffiths, C. Giannantonio, A. Hurley-Hanson, and E. Linstead, "Predicting Employer Recruitment of Individuals with Autism Spectrum Disorders with Decision Trees," Proc. 17th IEEE Int. Conf. Mach. Learn. Appl. ICMLA 2018, pp. 13661370, 2019

[18] M. B. Usta et al., "Use of machine learning methods in prediction of short-term outcome in autism spectrum disorders," Psychiatry Clin. Psychopharmacol., vol. 0573, 2018.

[19] V. P. Sudha and M. S. Vijaya, Machine Learning-Based Model for Identification of Syndromic Autism Spectrum Disorder, vol. 771. Springer Singapore, 2019.

[20] L. Bishop-Fitzpatrick et al., "Using machine learning to identify patterns of lifetime health problems in decedents with autism spectrum disorder," Autism Res., vol. 11, no. 8, pp. 1120-1128, 2018.

[21] D. Bone, S. L. Bishop, M. P. Black, M. S. Goodwin, C. Lord, and S. S. Narayanan, "Use of machine learning to improve autism screening and diagnostic instruments: effectiveness, efficiency, and multi-instrument fusion," J. Child Psychol. Psychiatry Allied Discip., vol. 57, no. 8, pp. 927-937, 2016.

[22] W. Liu, M. Li, and L. Yi, "Identifying children with autism spectrum disorder based on their face processing abnormality: A machine learning framework," Autism Res., vol. 9, no. 8, pp. 888898, 2016.

[23] S. Levy, M. Duda, N. Haber, and D. P. Wall, "Sparsifying machine learning models identify stable subsets of predictive features for behavioral detection of autism," Mol. Autism, vol. 8, no. 1, pp. 117, 2017.

[24] A. Crippa et al., "Use of Machine Learning to Identify Children with Autism and Their Motor Abnormalities," J. Autism Dev. Disord., vol. 45, no. 7, pp. 2146-2156, 2015.

[25] G. Wan et al., "Applying Eye Tracking to Identify Autism Spectrum Disorder in Children," J. Autism Dev. Disord., vol. 49, no. 1, pp. 209-215, 2019.

[26] A. Retico et al., "The effect of gender on the neuroanatomy of children with autism spectrum disorders: A support vector machine case-control study," Mol. Autism, vol. 7, no. 1, pp. 1-20, 2016.

[27] F. Thabtah, N. Abdelhamid, and D. Peebles, "A machine learning autism classification based on logistic regression analysis," Heal. Inf. Sci. Syst., vol. 7, no. 1, p. 12, 2019. 
Table 1. Results of Research Articles

\begin{tabular}{|c|c|c|c|c|c|c|c|c|c|}
\hline \multirow[t]{2}{*}{ No } & \multirow[t]{2}{*}{ Data } & \multirow[t]{2}{*}{ Samples } & \multirow{2}{*}{$\begin{array}{c}\text { Machine } \\
\text { Learning } \\
\text { Method Used }\end{array}$} & \multirow{2}{*}{$\begin{array}{l}\text { Predicti } \\
\text { on Goal }\end{array}$} & \multicolumn{4}{|c|}{ Result } & \multirow[t]{2}{*}{ Citation } \\
\hline & & & & & Specificity & Sensitivity & Accuracy & $\begin{array}{l}\text { Area } \\
\text { Under } \\
\text { Curve } \\
\text { (AUC) }\end{array}$ & \\
\hline 1 & $\begin{array}{l}65 \text { item social } \\
\text { responsiveness } \\
\text { scale score } \\
\text { sheet }\end{array}$ & 2925 & $\begin{array}{c}\text { Decision tree, } \\
\text { Random forest, } \\
\text { Support vector } \\
\text { classification } \\
\text { (SVM), } \\
\text { Logistic, } \\
\text { Regression, } \\
\text { categorical } \\
\text { Lasso, Linear } \\
\text { Discriminant } \\
\text { Analysis } \\
\end{array}$ & $\begin{array}{l}\text { ASD/A } \\
\text { DHD }\end{array}$ & - & - & - & 0.965 & [8] \\
\hline 2 & Survey & 263 employers & Decision tree & ASD & $82 \%$ & - & $75 \%$ & - & {$[17]$} \\
\hline 3 & $\begin{array}{c}\text { The Autism } \\
\text { and } \\
\text { Developmental } \\
\text { Disabilities } \\
\text { Monitoring } \\
\text { (ADDM) }\end{array}$ & 601 Children & Random Forest & ASD & $89.2 \%$ & $8 ., 0 \%$ & - & - & {$[12]$} \\
\hline 4 & $\begin{array}{l}\text { Blood-based } \\
\text { Gene } \\
\text { Expression } \\
\text { Signature }\end{array}$ & 21 Adult & $\begin{array}{c}\text { SVM, KNN, } \\
\text { Linear } \\
\text { Discriminant } \\
\text { analysis (LDA) }\end{array}$ & ASD & $\begin{array}{l}87.5 \% \\
\text { (SVM, } \\
\text { KNN) }\end{array}$ & $\begin{array}{l}100 \% \\
\text { (SVM, } \\
\text { KNN) }\end{array}$ & $\begin{array}{l}93.8 \% \\
(\mathrm{SVM}, \\
\text { KNN) }\end{array}$ & - & [7] \\
\hline 5 & ADI-R, SRS & $\begin{array}{l}1264 \text { ASD and } \\
462 \text { verbal } \\
\text { individuals with } \\
\text { non-ASD } \\
\text { developmental } \\
\text { or psychiatric } \\
\text { disorders }\end{array}$ & SVM & $\begin{array}{l}\text { ASD/N } \\
\text { on ASD }\end{array}$ & $\begin{array}{l}59.0 \% \text {, and } \\
53.4 \%\end{array}$ & $\begin{array}{c}89.2 \% \text {, and } \\
86.7 \%\end{array}$ & - & - & [21] \\
\hline 6 & $\begin{array}{c}\text { ICD, V-Codes, } \\
\text { E'Codes }\end{array}$ & $\begin{array}{l}91 \mathrm{ASD}, 6186 \\
\text { control }\end{array}$ & Random Forest & $\begin{array}{l}\text { ASD/Ty } \\
\text { pically } \\
\text { Develop } \\
\text { ing } \\
\text { (TD) }\end{array}$ & $94 \%$ & $75 \%$ & $93 \%$ & 0,88 & [20] \\
\hline 7 & $\begin{array}{c}\text { Face } \\
\text { recognition } \\
\text { task }\end{array}$ & $\begin{array}{c}29 \text { ASD, } 29 \text { TD } \\
\text { (Typically } \\
\text { Developing) }\end{array}$ & SVM & $\begin{array}{l}\text { ASD/T } \\
\text { D } \\
\text { (Typical } \\
\text { ly } \\
\text { Develop } \\
\text { ing) } \\
\end{array}$ & $86 \%$ & $93 \%$ & $88 \%$ & $89 \%$ & {$[22]$} \\
\hline 8 & Kinematic data & $\begin{array}{c}16 \text { ASD, } 14 \text { TD } \\
\text { (Typically } \\
\text { Developing) }\end{array}$ & $\begin{array}{c}\text { SVM, Random } \\
\text { Forest, Naïve } \\
\text { Bayes, Decision } \\
\text { Tree }\end{array}$ & $\begin{array}{l}\text { ASD/T } \\
\text { D } \\
\text { (Typical } \\
\text { ly } \\
\text { Develop } \\
\text { ing) } \\
\end{array}$ & $87.5 \%$ & $85.7 \%$ & $867 \%$ & - & [14] \\
\hline 9 & $\begin{array}{c}\text { ADOS } \\
\text { (Autism } \\
\text { Diagnostic } \\
\text { Observation } \\
\end{array}$ & $\begin{array}{l}\text { Module } 2 \text { for } \\
\text { children with } \\
\text { phrased speech } \\
\text { (1319 ASD, } 70\end{array}$ & $\begin{array}{l}\text { SVM linear and } \\
\text { Logistic } \\
\text { Regression }\end{array}$ & $\begin{array}{l}\text { ASD/N } \\
\text { on ASD }\end{array}$ & - & - & - & $\begin{array}{c}0.93 \\
\text { Module } 2 \\
\text { and } 0.95 \\
\text { module } 3 \\
\end{array}$ & [23] \\
\hline
\end{tabular}




\begin{tabular}{|c|c|c|c|c|c|c|c|c|c|}
\hline & $\begin{array}{l}\text { Schedule) } \\
\text { Module } 2 \text { and } \\
\text { Module } 3\end{array}$ & $\begin{array}{c}\text { Control). } \\
\text { Module } 3 \text { for } \\
\text { children with } \\
\text { verbal } \\
\text { fluency }(2870 \\
\text { ASD, } 273 \\
\text { control }\end{array}$ & & & & & & & \\
\hline 10 & $\begin{array}{c}\text { Autism } \\
\text { Behavior } \\
\text { Checklist, } \\
\text { Aberrant } \\
\text { Behavior } \\
\text { Checklist, } \\
\text { Clinical } \\
\text { Global } \\
\text { Impression } \\
\text { scales at } \\
\text { baseline (T0) } \\
\text { and 12th (T1), } \\
\text { 24th (T2), } \\
\text { and 36th (T3) } \\
\text { months }\end{array}$ & $\begin{array}{l}433 \text { children } \\
\text { with ASD }\end{array}$ & $\begin{array}{c}\text { Naïve Bayes, } \\
\text { Generalized } \\
\text { Linear Model, } \\
\text { Logistic } \\
\text { Regression, and } \\
\text { Decision Tree }\end{array}$ & ASD & $\begin{array}{c}19.4 \%, \\
54.8 \% \text {, } \\
54.8 \% \text { and } \\
61.3 \%\end{array}$ & $\begin{array}{c}95.2 \% \\
80.5 \%, \\
85.7 \% \text { and } \\
81.1 \%\end{array}$ & $\begin{array}{c}50.0 \%, \\
69.2 \%, \\
67.3 \% \\
\text { and } \\
71.2 \%\end{array}$ & $\begin{array}{c}0.696, \\
0.668, \\
0.665 \\
\text { and } \\
0.707\end{array}$ & [18] \\
\hline 11 & $\begin{array}{c}\text { ADOS } \\
\text { (Module } 2 \text { and } \\
\text { module } 3 \text { ) }\end{array}$ & $\begin{array}{c}4540 \\
\text { individuals }\end{array}$ & $\begin{array}{c}\text { ADTree, } \\
\text { Functional tree, } \\
\text { Lib SVM, LMT, } \\
\text { Logistic } \\
\text { Regression, } \\
\text { Naïve Bayes, } \\
\text { NB Tree, } \\
\text { Random Forest }\end{array}$ & ASD & $\begin{array}{c}89.39 \% \\
\text { (Logistic } \\
\text { Regression } \\
\text { ) } \\
97 \% \\
(\mathrm{SVM})\end{array}$ & $\begin{array}{c}98.81 \% \\
\text { (Logistic } \\
\text { Regression) } \\
97 \%(\mathrm{SVM})\end{array}$ & $\begin{array}{c}98.27 \% \\
\text { and } \\
97.66 \%\end{array}$ & - & [5] \\
\hline 12 & $\begin{array}{l}152 \text { structural } \\
\text { MRI scan }\end{array}$ & $\begin{array}{c}76 \text { young } \\
\text { children with } \\
\text { ASD ( } 38 \text { males } \\
\text { and } 38 \text { females) }\end{array}$ & SVM & ASD & - & - & - & - & [26] \\
\hline 13 & $\begin{array}{c}\text { Autistic } \\
\text { quotient )AQ) }\end{array}$ & $\begin{array}{l}\text { AQ } 10 \text { adult } \\
\text { and AQ } 10 \\
\text { adolescent }\end{array}$ & $\begin{array}{c}\text { Logistic } \\
\text { Regression }\end{array}$ & ASD & $82.64 \%$ & $85.90 \%$ & $85.88 \%$ & - & {$[27]$} \\
\hline 14 & Aye tracking & $\begin{array}{c}37 \text { with ASD } \\
\text { and } 37 \text { typically } \\
\text { developing } \\
\text { (TD) }\end{array}$ & SVM & $\begin{array}{c}\text { ASD } \\
\text { and Non } \\
\text { ASD }\end{array}$ & $83.8 \%$ & $86.5 \%$ & $85.1 \%$ & - & [25] \\
\hline 15 & $\begin{array}{l}\text { Kinematic } \\
\text { analysis }\end{array}$ & $\begin{array}{l}15 \text { preschool } \\
\text { children with } \\
\text { ASD from } \\
\text { 15typically } \\
\text { developing }\end{array}$ & SVM & ASD & $93.8 \%$ & $100 \%$ & $96.7 \%$ & - & [24] \\
\hline 16 & $\begin{array}{c}\text { Gene } \\
\text { sequences }\end{array}$ & - & $\begin{array}{l}\text { Decision Tree } \\
\text { SVM and } \\
\text { Multilayer } \\
\text { perceptron }\end{array}$ & ASD & $\begin{array}{c}(99 \% \\
\text { Decision } \\
\text { Tree) } \\
\\
(\text { SVM } \\
98 \%) \text { and } \\
\text { (MLP } \\
99 \%)\end{array}$ & - & $\begin{array}{c}(98 \% \\
\text { decision } \\
\text { tree) } \\
\\
(\mathrm{SVM} \\
96 \%) \text { and } \\
(\mathrm{MLP} \\
95 \%)\end{array}$ & - & [19] \\
\hline
\end{tabular}




\section{CERTIFICATE}

This certificate is presented to

\section{Mr. Andi Wahju Rahardjo Eman-} uel

\section{as}

for outstanding contribution in the 4th 2019 International Conference on Information Technology, Information System, and Electrical Engineering held in Yogyakarta, Indonesia on 20 - 21 November 2019

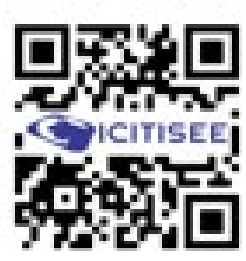

Conference No. \#48480

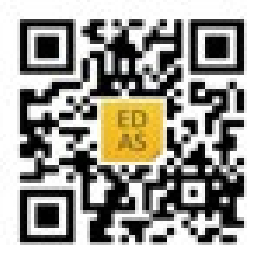

ID number 1129597

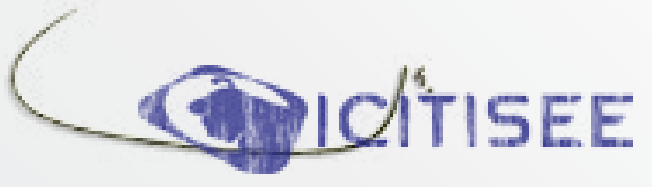

Dr. Kusrini, M.Kom.

General Chair

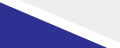

- IEEE INDONESIA SECTION
Department of mation Engineering Universitas Gadjah Mada 